\title{
Hafnium-Silicon Precipitate Structure Determination in a New Heat-Resistant Ferritic Alloy by Precession Electron Diffraction Techniques
}

\author{
Désirée Viladot, ${ }^{1}$ Joaquim Portillo, ${ }^{2,3}$ Mauro Gemí, ${ }^{4}$ Stavros Nicolopoulos, ${ }^{3}$ and \\ Núria Llorca-Isern ${ }^{1, \star}$ \\ ${ }^{1}$ Departament de Ciència de Materials i Enginyeria Metal.lúrgica, Facultat de Química, Universitat de Barcelona, \\ C/Martí i Franquès 1-11, Barcelona 08028, Spain \\ ${ }^{2}$ Centres Cientifics i Tecnologics (CCiT), Universitat de Barcelona/Solé i Sabaris 1-3, Barcelona 08028, Spain \\ ${ }^{3}$ NanoMEGAS, Boulevard Edmond Machtens 79, Brussels B-1080, Belgium \\ ${ }^{4}$ Center for Nanotechnology Innovation@NEST, Istituto Italiano di Tecnologia, Pisa 56127, Italy
}

\begin{abstract}
The structure determination of an $\mathrm{HfSi}_{4}$ precipitate has been carried out by a combination of two precession electron diffraction techniques: high precession angle, $2.2^{\circ}$, single pattern collection at eight different zone axes and low precession angle, $0.5^{\circ}$, serial collection of patterns obtained by increasing tilts of $1^{\circ}$. A threedimensional reconstruction of the associated reciprocal space shows an orthorhombic unit cell with parameters $a=11.4 \AA, b=11.8 \AA, c=14.6 \AA$, and an extinction condition of $(h k l) h+k$ odd. The merged intensities from the high angle precession patterns have been symmetry tested for possible space groups (SG) fulfilling this condition and a best symmetrization residual found at 18\% for SG $65 \mathrm{Cmmm}$. Use of the SIR2011 direct methods program allowed solving the structure with a structure residual of $18 \%$. The precipitate objects of this study were reproducibly found in a newly implemented alloy, designed according to molecular orbital theory.
\end{abstract}

Key words: precession electron diffraction, precession-assisted tomographic diffraction, crystal structure determination, hafnium-silicon precipitate

\section{INTRODUCTION}

Modern materials science and engineering require strong knowledge of the investigated structures and their properties. X-ray diffraction (XRD) is the most common method used in structural analysis. However, when dealing with submicron size precipitates, limitations of the technique including peak broadening because of crystal form factor and strong matrix signal overlap in case of embedded precipitates in a thin foil are critical drawbacks for structure determination. Consequently, when transmission electron microscopy (TEM) transparent thin foils were prepared and unknown submicron precipitates were first observed, XRD was disregarded as a means of identification of their structure. The microstructure of the studied sample as revealed in the TEM consists of a polycrystalline iron ferritic matrix with an interdendritic filling phase rich in dense elongated or round precipitates ranging from 50 to $300 \mathrm{~nm}$ thick.

Conventional electron diffraction may in principle lead to crystallographic structural information, provided the local sample thickness contributing to the diffraction pattern is $<50 \mathrm{~nm}$ at $200 \mathrm{kV}$. At this thickness, even medium atomic weight compounds may display a nonkinematic nature in the measured diffracted intensities, which is a great concern for reliable structure analysis. Quantitative exploitation of such diffraction data leading to a structure proposal has to

Received March 18, 2013; accepted September 12, 2013

${ }^{*}$ Corresponding author. E-mail: nullorca@ub.edu be confronted with simulations using dynamical diffraction calculations via multislice or Bloch wave propagation models for final structure assessment. The validity of the proposed structure is parameterized via a cumulative normalized structural residue, $R$, between measured and calculated intensities in the operative reflections (Sinkler \& Marks, 1999).

The use of a special diffraction technique first introduced by Vincent and Midgley in 1994 (Vincent \& Midgley, 1994) has seen widespread use within the electron microscopy community as a tool to help overcome these problems (Weirich et al., 2006). Precession electron diffraction (PED) consists of a two-stage modification of the electron beam paths, both above and below the specimen. Effectively, the beam is either focussed or kept quasi parallel on a region of interest (ROI) in the sample and rocked at a frequency of $100 \mathrm{~Hz}$ on the surface of an inverted cone, the vertex of which is located precisely at this ROI. The angle between the cone height-which coincides with the optical axisand the cone surface is called the precession angle, and the illumination mode thus produced inverted hollow-cone illumination (Otten, 1991). The beam path modification below the specimen implies counter-precessing both transmitted and diffracted trajectories with the help of the microscope column image deflection coils in order to produce a pseudo-stationary electron diffraction (ED) pattern, where spot intensities flicker at $100 \mathrm{~Hz}$ (Own et al., 2004).

PED shows three significant advantages over conventional stationary on-axis ED (Morniroli et al., 2007): (1) integrated intensities from observed PED reflections are 
usually closer to purely kinematical values due to the offzone axis condition that favors simultaneous few-beam excitations at a frozen moment in time, (2) PED patterns display a larger number of reflections than those arising from conventional diffraction patterns, and (3) depending on the precession angle chosen, if enough exposure time is used, a broadened FOLZ disc will be observed and the two-dimensional (2D) symmetry present therein is useful for space groups (SG) identification (Morniroli \& Ji, 2009). The first two advantages become visible even at moderate precession angles of $0.4-0.8^{\circ}$, whereas the last one requires precession angle values from $2.0^{\circ}$ upwards.

In the present study, the crystallographic structure of a repetitive unknown precipitate in a new iron-based heatresistant alloy, which displays improved properties, has been solved using only quantitative information from low- and high-angle PED patterns together with energy-dispersive $\mathrm{X}$-ray (EDS) semi-quantitative microanalysis. The particular microstructure observed in the alloy is responsible for its interesting creep and corrosion behavior at high temperatures $\left(800-1,200^{\circ} \mathrm{C}\right)$. After conventional TEM investigation of the sample, 50-300 nm sized hafnium-silicon (Hf-Si) precipitates were located in the interdendritic regions. The determination of their crystallographic structure is the goal of this work.

The originality of this structural study lies in the fact that for the first time a submicron structural determination is obtained in the TEM alone (no XRD data is possible from these precipitates) without high-resolution imaging (unavailable because of the magnetic nature of the sample), with only in-column EDS microanalysis and two PED techniques: low PED angle partial 3D tomographic reconstruction of reciprocal space and high PED angle quantitative diffraction analysis on a reduced group of zone-axis orientations. It is worthwhile to point out that some nanometersized crystal structures, usually observed in powders dispersed on a holey carbon grid, have already been solved via a quasi-complete tomographic 3D reconstruction of the crystal reciprocal space using data obtained at $\pm 60^{\circ}$ holder tilts (where only a missing cone of reflections is lacking) (Kolb et al., 2011). This procedure, however, is only partially available in the case of magnetic foil-type samples, due to the extreme difficulty of precession and counter-precession signal adjustment for high holder-tilt angles, when the interaction between the objective lens field and that of the sample is nonnegligible. We have therefore used here, for the first time, a complementary approach of: (a) partial (holder-tilt angle limited to $\pm 15^{\circ}$ ) low precession angle, $0.5^{\circ}$, PED reciprocal space $3 \mathrm{D}$ reconstruction and (b) high precession angle, $2.2^{\circ}$, PED pattern quantitative analysis on a few selected zone-axis directions with SIR2011 (Burla et al., 2012).

\section{Materials and Methods}

The PED experiments were carried out both on a JEOL JEM $2100 \mathrm{LaB}_{6}$ (JEOL Ltd., Tokyo, Japan) operating at $200 \mathrm{kV}$ for large angle, $2.2^{\circ}$, precession zone-axis pattern collection and on a LIBRA120KV instrument for the low precession angle, $0.5^{\circ}$, serial precession off-zone axis tomographic manual pattern collection, leading to $3 \mathrm{D}$ reconstruction of the reciprocal space associated with the precipitates. Both electron transmission microscopes were equipped with the commercial precession system DigiSTAR manufactured by NanoMEGAS. The device may obtain PED patterns of varying precession angles, from 0 to $2.5^{\circ}$, with easy counterprecession adjustment and is readily attachable to both TEM microscopes by storing the instrument-dependent precession and counterprecession alignment files in the memory of the computer in control of the precession signal generator, Nanomegas P1000 unit. The diffraction images were recorded with an Orius Multiscan CCD camera by Gatan (Pleasanton, CA, USA) and the X-ray maps and spectra with an X-ray microanalysis INCA ultrathin window $\mathrm{Si}(\mathrm{Li})$ detector by Oxford Instruments (Abington, Oxfordshire, UK). Sample preparation included use of a Gatan precision ion-polishing system Model 691 PIPS.

The new alloy RFT is a Fe-based alloy containing chromium (Cr), aluminum (Al), and nickel (Ni), and minority amounts of silicon $(\mathrm{Si})$, yttrium $(\mathrm{Y})$, and hafnium (Hf) in $<1 \mathrm{wt} \%$. The precipitates for this study were formed during the cooling of the samples in an electric arc furnace Arc Melter AM by Edmund Bühler, GmbH (Hechingen, Germany). TEM samples were prepared by diamond saw slicing, mechanical parallel polishing to $1 \mathrm{~mm}$ thickness, and disc punching on the obtained slices measuring $3 \mathrm{~mm}$ in diameter. A second mechanical parallel polishing further thinned them to $\sim 0.15 \mathrm{~mm}$ in thickness and finally the center was dimpled to a local thickness of $30 \mathrm{~mm}$. The samples were then argon ion polished from both sides at $4 \mathrm{kV}$ acceleration voltage, using high flux and combining an initial incidence milling angle of $7^{\circ}$ with a finishing low milling angle of $3^{\circ}$ to reach central perforation.

The sample was first observed on the 200KV JEOL to obtain EDS microanalysis mappings and spectra, as well as high precession angle $2.2^{\circ} \mathrm{ED}$ patterns on nine low symmetry zone axes observable on the precipitates. This high precession angle allows intensity extraction via software with a greater approach to kinematic conditions than lower angle values. Thereafter, the sample was observed on a $120 \mathrm{KV}$ Libra ZEISS instrument (Carl Zeiss, Oberkochen, Germany) to record a tomographic series of $31 \mathrm{ED}$ patterns from the same precipitate. The serial acquisition was started from a nonzone axis condition and acquired manually at sample holder-tilting intervals of $1^{\circ}$ with precession illumination active at $0.5^{\circ}$ precession angle. This low precession angle, although limited for quantitative processing of intensities present in operative reflections, allows nonoverlapping conditions of successively recorded ED patterns and is, therefore, a convenient choice for a partial 3D reconstruction of the reciprocal lattice associated to the precipitates (Gorelik et al., 2011). From this partial reciprocal space reconstruction, a precise determination of the crystal system, its lattice parameters, and the applicable extinction conditions 

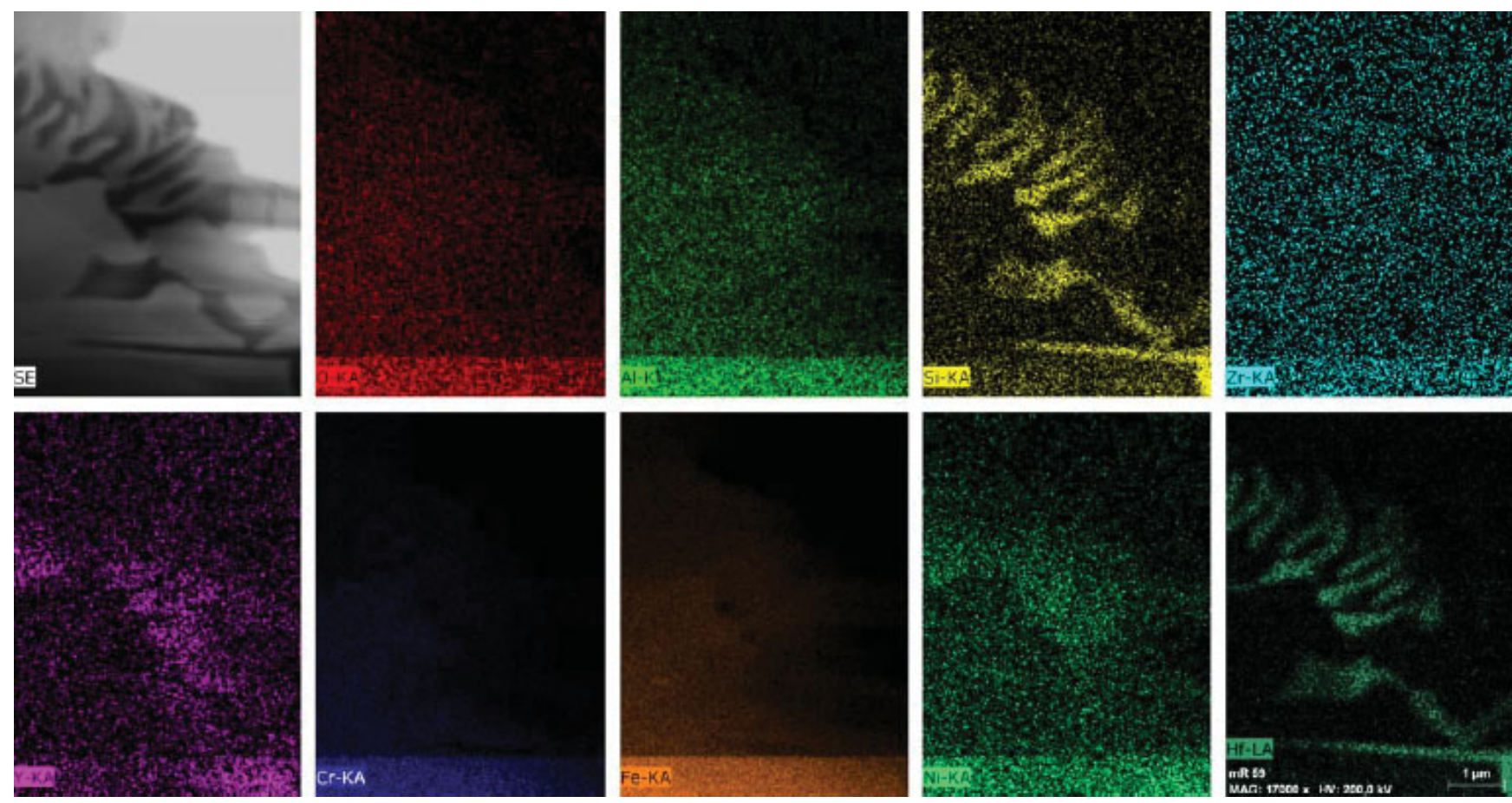

Figure 1. Interdendritic zone energy-dispersive $\mathrm{X}$-ray map distribution (from left to right) of O K (red), $\mathrm{Al} \mathrm{K}$ (green), Si $\mathrm{K}$ (yellow), Zr K $\alpha$ (light blue), Y K $\alpha$ (purple), Fe K $\alpha$ (dark blue), Cr K $\alpha$ (orange), Ni K $\alpha$ (light green), Hf L $\alpha$ (dark green). Precipitate intensity reveals Hf-Si content, whereas the interdendrite phase is rich in Y and Ni.

present in the operative reflections has been possible. This determination was introduced in the PHIDO procedure from CRISP version 2.1a (www.calidris-em.com, 2004) for precise indexation. The intensities extracted from the indexed PED were symmetrized and merged by TRIPLE version 2.0a. The resultant merged intensities were used to solve the structure using SIR2011 version 1.0.1. These data were treated by direct methods. Triple relations were used to form triplet series with a common reflection and the phase was deduced from the so-called tangent formula (Zou et al., 2012).

The alloy contains three well-distinguished phases: matrix, interdendritic, and Hf-Si precipitates embedded in the latter. Matrix composition is mainly formed by $\mathrm{Fe}, \mathrm{Cr}$, and $\mathrm{Al}$, and shows a bcc type lattice; moreover, it displays a weak magnetic behavior that hinders an automatized procedure of the serial PED data collection in the LIBRA $120 \mathrm{KV}$ instruments. The interdendritic phase concentrates the $\mathrm{Y}$ of the composition together with $\mathrm{Ni}$ and $\mathrm{Hf}$, but is not the subject of the present study.

\section{Results AND Discussion}

The three well-differentiated morphological phases in the alloy may be seen in the TEM micrographs of Figure 1 (top left). In Figure 1, the EDS mapping shows the Hf and Si content of relatively large precipitates. The semi-quantitative treatment of Hf L $\alpha$ (dark green) and Si K $\alpha$ (yellow) emission peaks in the thin foil approximation using CliffLorimer normalization $k$-factors embedded in the INCA software leads to a Hf 1:Si 4 atomic ratio, therefore to the stoichiometric formula $\mathrm{HfSi}_{4}$.

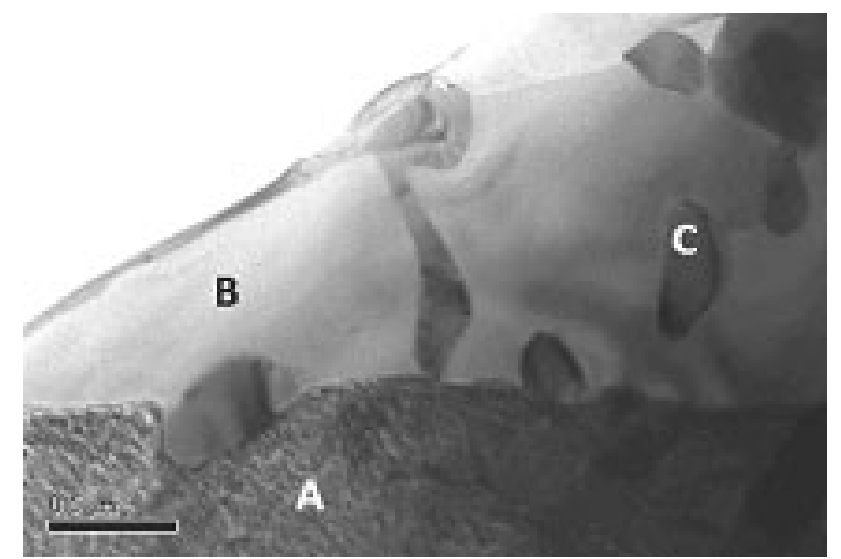

Figure 2. Left: transmission electron microscopy bright field micrograph of interdendritic zone and (at the bottom, marked A) ferritic grain $\langle 100\rangle$ oriented. A: bcc Fe matrix with $a=2.82 \AA$, B: interdendritic phase, and C: HfSi precipitate. Right: $2.2^{\circ}$ precession electron diffraction overlay including the three phases present in the alloy.

In Figure 2, the different features are marked as A, B, and $\mathrm{C}$, with $\mathrm{A}$ being the alloy ferritic matrix, $\mathrm{B}$ the interdendritic filling phase, and $\mathrm{C}$ the $\mathrm{HfSi}_{4}$ precipitates.

The smallest selected-area aperture was chosen to isolate PED patterns from each of the individual three phases and these were recorded with precession angles of $0^{\circ}$ and $2.2^{\circ}$. Figure 3 shows the nine zone axes used for the precipitate identification.

PED tomography was performed on one of the precipitates to obtain a representative sampling of the $3 \mathrm{D}$ recipro- 

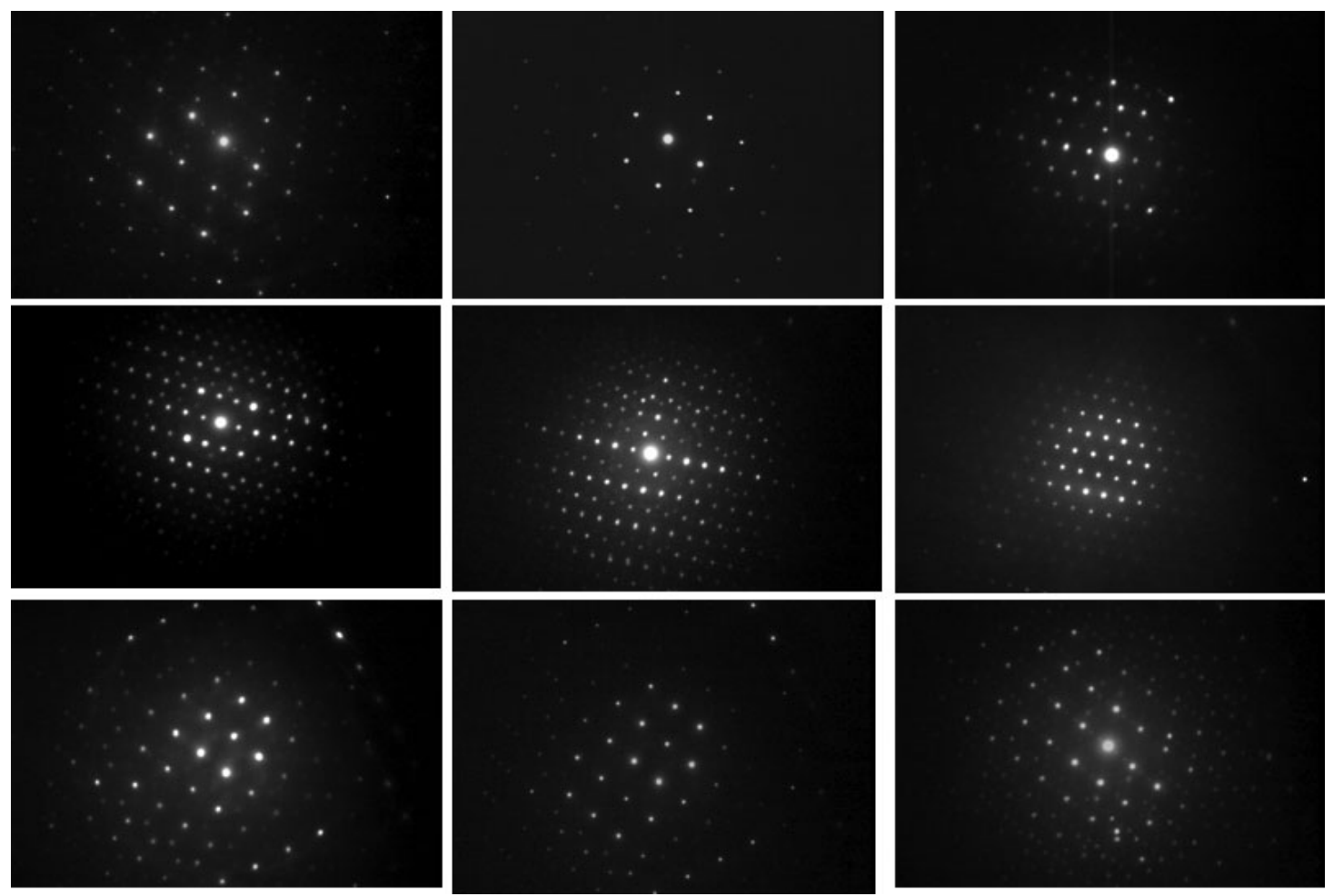

Figure 3. Non-precession electron diffraction (PED) (left) and PED (right) diffraction patterns from matrix (top), interdendritic solid solution phase (centre) and HfSi precipitate (bottom).

cal space. Due to the ferritic phase, the specimen showed some magnetization that hindered a complete data collection for full reconstruction, since as the specimen was tilted, the beam shifted on the sample plane and also in the back focal plane of the objective lens. However, the collected data from 31 patterns was sufficient to be analyzed with the PETS software (Lucas Palatinus, 2011), a data processing program for analysis of ED data from the Institute of Physics of the AS CR (Prague). The reconstruction in three dimensions allowed exploration of reciprocal space and the reconstructed volume was indexed by PETS with the indexing tool of the JANA program (Petricek et al., 2006) as an orthorhombic lattice with $a=11.4 \AA, b=11.8 \AA, c=$ $14.6 \AA$. A projection of the reconstructed reciprocal volume along [001] and [010] clearly showed the reflection visibility conditions $h k l, h+k=2 n$ (Fig. 4).

Using this information on crystal system and extinction conditions, only a limited number of SG are compatible with the orthorhombic crystal system and extinction ( $h k l) h+k$ odd, namely: C222 1 (20), C222 (21), Cmm2 (35), $C m c 2_{1}$ or $C c m 2_{1}$ (36), Ccc2 (37), Cm2 $2 m$ or $C 2 m m$ (38), Cm2e (39), C2cm or Cc2m (40), Cc2e (41), Cmcm or Ccmm (63), Ccme (64), Cmmm (65), Cccm (66), Cmme (67), Ccce (68).

Once the precipitate cell parameters and possible SG have been determined, reflection intensities have been extracted from the nine different zone-axis high-angle PED patterns using the ELD program of the CRISP 2.1a package (Calidris, http://www.calidris-em.com), as may be seen in Figure 5.

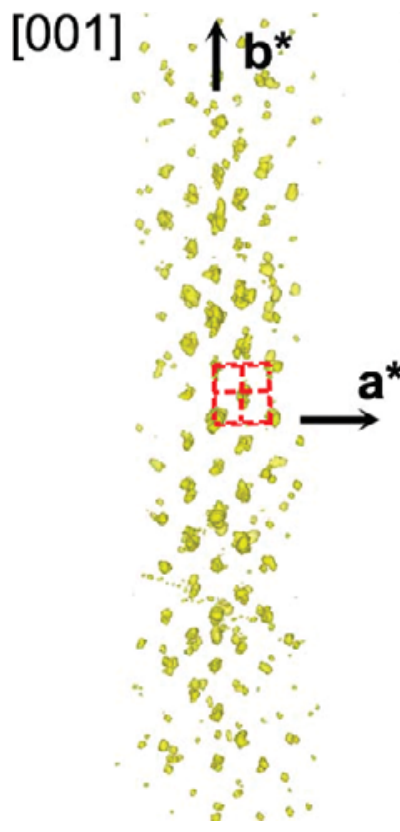

[010]

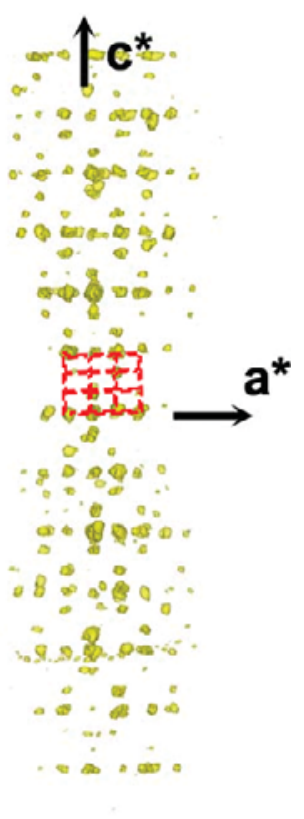

Figure 4. Reconstructed reciprocal space of one crystal grain of the precipitates. The projections along two main crystallographic directions are displayed. In the [001] projection extinction is clearly visible.

Accurate indexing is obtained with PHIDO with a base vectors tolerance kept low (strictly $2 \%$ tolerance for $u$ and $v$ and $4 \%$ for their relative angle). Nine single PED patterns were saved imposing a $d$-spacing value cut-off of $0.5 \AA$. Concerning zone axis [ $\left[\begin{array}{lll}-5 & 25 & 2\end{array}\right]$, it is the exact value found 


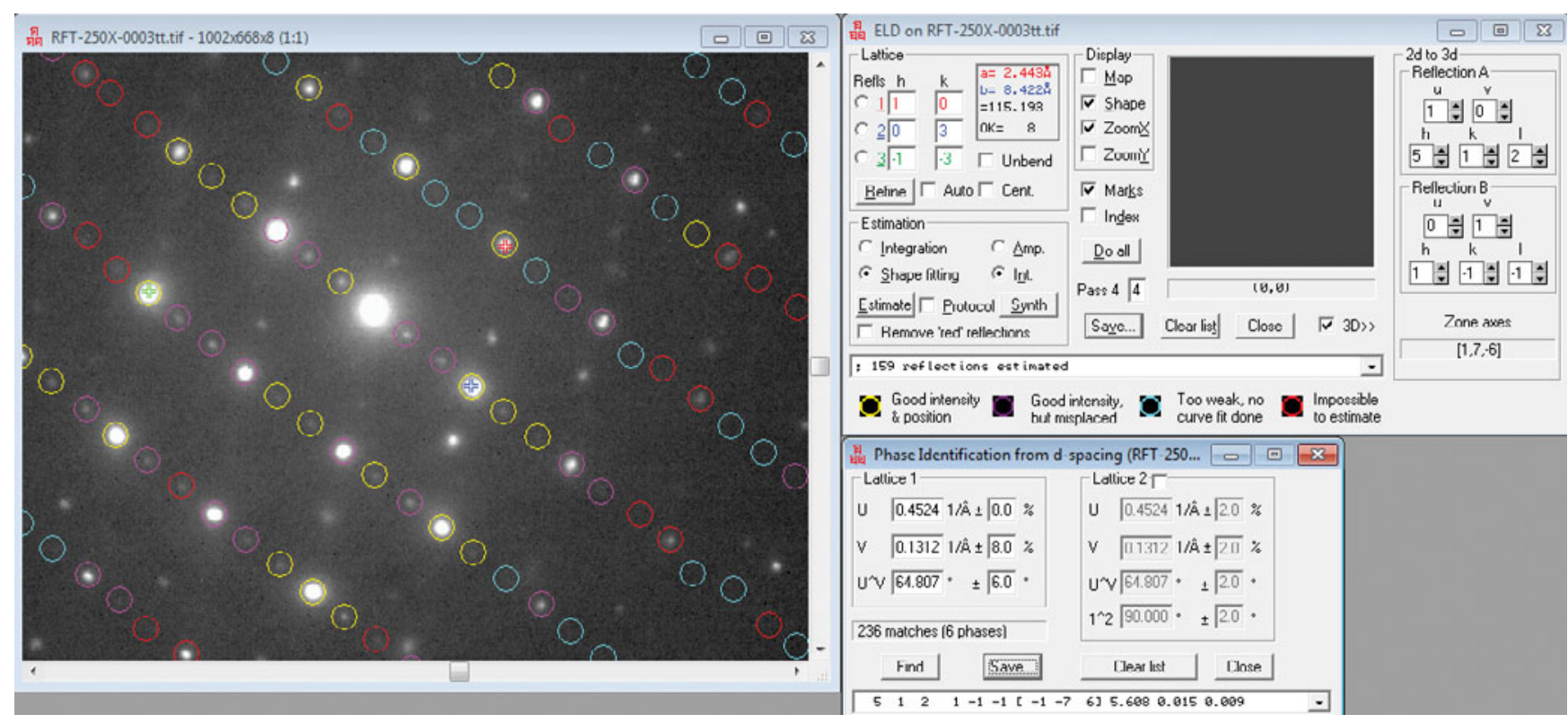

Figure 5. Intensity extraction from $2.2^{\circ}$ precessed zone axis $\langle 17-6\rangle$, using ELD software from Calidris.

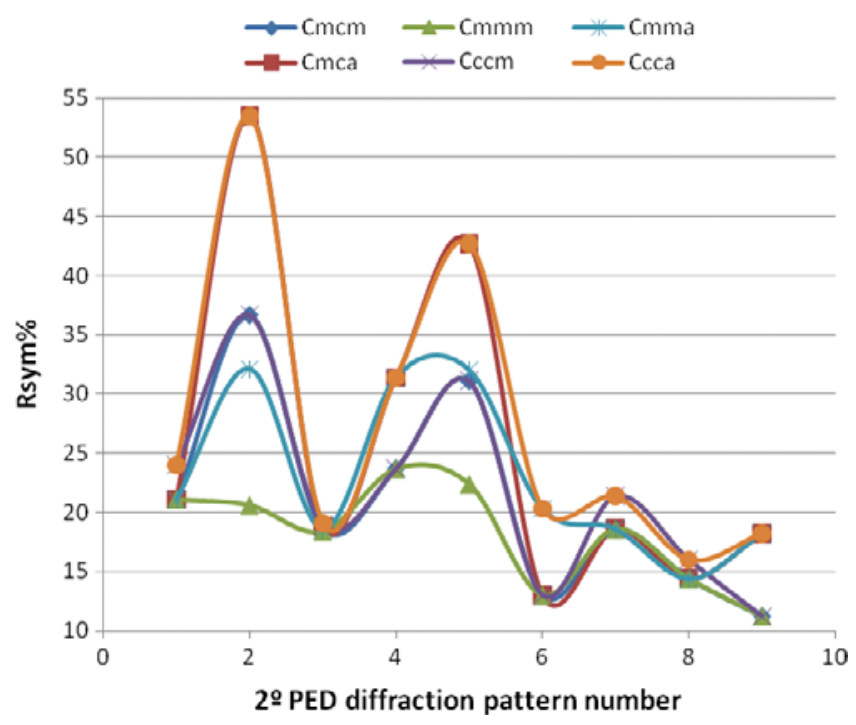

Figure 6. Space groups symmetrization residuals obtained for each precession electron diffraction pattern.

Table 1. Determined Pattern Zone Axes.

\begin{tabular}{lrc}
\hline $\begin{array}{l}\text { Diffraction } \\
\text { Pattern }\end{array}$ & $\begin{array}{c}\text { Pattern } \\
\text { Indexation }\end{array}$ & \%Rsym \\
\hline 1 & {$[2 \overline{3} 2]$} & 21.1 \\
2 & {$[\overline{5} 252]$} & 20.6 \\
3 & {$[1 \overline{\overline{6}}]$} & 18.4 \\
4 & {$[\overline{1} \overline{1} 3]$} & 23.7 \\
5 & {$[\overline{5} 252]$} & 22.4 \\
6 & {$[\overline{1} \overline{1} 3]$} & 13 \\
7 & {$[2 \overline{3} 2]$} & 18.6 \\
8 & {$[2 \overline{3} 2]$} & 14.4 \\
9 & {$[5 \overline{1} \overline{1}]$} & 11.2 \\
\hline
\end{tabular}

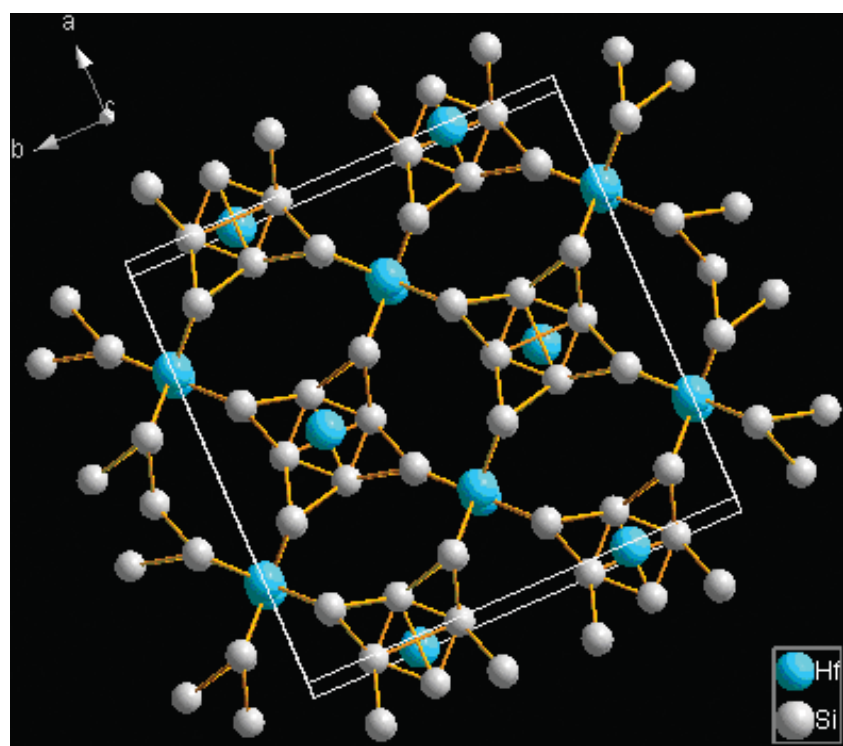

Figure 7. HfSi precipitates structural simulation (SIR2011).

when taking into account all recorded spots on the $2^{\circ}$ precessed pattern. The low Miller index axis to which it may be approximated is $\left[\begin{array}{lll}-2 & 9 & 1\end{array}\right]$ by $2^{\circ}$. This is possibly the zone axis which one may determine if recording the pattern without precession, due to the fact that the outermost operative reflections would not be active and it is precisely these which allow a more precise determination of the direction in real space to which the recorded diffraction pattern is perpendicular. Since our data are high PED angle diffraction data, we have used them (central and outer most noncentral diffraction spots) to determine the zone axis as accurately as possible (Table 1).

All PED patterns were symmetrized with all the possible SG and symmetrization residuals were compared to find 
Table 2. Atomic Position Values.

\begin{tabular}{lccccccccc}
\hline Name & Type & Serial & $x$ & $y$ & $z$ & Occ & B(iso) & U(iso) & Height ${ }^{*} 10$ \\
\hline Hf1 & hf & 1 & 0.7559 & 0.0000 & 0.0803 & 0.5000 & 1.5600 & 0.0198 & 52 \\
Hf2 & hf & 2 & 0.0000 & 0.0000 & 0.0000 & 0.1250 & 0.0220 & 0.0003 & 37 \\
Si1 & si & 1 & 0.0000 & 0.0761 & 0.1968 & 0.5000 & 1.6170 & 0.0205 & 26 \\
Si2 & si & 2 & 0.7500 & 0.2500 & 0.4359 & 0.5000 & 0.3040 & 0.0039 & 26 \\
Si3 & si & 3 & 0.8031 & 0.1568 & 0.5000 & 0.5000 & 2.2870 & 0.0290 & 20 \\
Si4 & si & 2 & 0.8351 & 0.8542 & 0.0000 & 0.5000 & 1.1640 & 0.0147 & 20 \\
Si5 & si & 6 & 0.9056 & 0.2704 & 0.2243 & 1.0000 & 0.7480 & 0.0095 & 16 \\
Si6 & si & 4 & 0.0000 & 0.1417 & 0.4051 & 0.5000 & 1.3990 & 0.0177 & 15 \\
Si7 & si & 5 & 0.6473 & 0.0000 & 0.2480 & 0.5000 & 0.0530 & 0.0007 & 14 \\
Si8 & si & 6 & 0.5000 & 0.0877 & 0.4353 & 0.5000 & 13.7700 & 0.1744 & 7 \\
\hline
\end{tabular}

out the SG that provides the lowest residual. Some of these residuals are represented in Figure 6.

With the list of extracted and symmetrized intensities from these nine low symmetry zone axes obtained at $2.2^{\circ}$ precession angle at $200 \mathrm{kV}$, a unique $(h k l)$ file was created through merging with Calidris Triple software by means of common reflection scale factor renormalization. This consists of a progressive paired comparison of the indexed PED patterns, detecting those reflections which appear on both compared patterns; for each common reflection, a normalization factor is calculated and, since there usually is a list of common reflections to each pair of patterns, one is confronted with a list of normalization factors, which may be filtered according to intensity or distance to produce an average normalization factor. The lowest common residual is obtained for SG 65, Cmmm, as may be observed in Figure 6, with a medium value of $18 \%$ (Rauch et al., 2008). The merged file exhibited a data set of 255 independent reflections.

Next, space group Cmmm was selected for solving the structure by direct methods with the program SIR2011 (Gemmi et al., 2002). The most probable solution with these data by SIR20011 gave an $R$-factor of $28.08 \%$.

Although the residual is low, $28.08 \%$ for the highest figure of merit, one has to look for the plausibility of the structure proposed from the crystal chemistry. For example, if no neighboring $\mathrm{Hf}$ atoms were found, the coordination polyhedron for each $\mathrm{Hf}$ atom with $\mathrm{Si}$ atom has plausible distances and conforms a tetrahedron.

Atom position list should be edited, ghosts removed, and Hf and Si peaks with Debye-Waller negative factors B probably also removed. Figure 7 shows the simulation of the precipitate structure.

Table 2 lists the position values of all the different atoms situated in the unit cell.

Kinematical simulation of the [232] indexed pattern according to the lattice parameters and SG determination as provided from the tomographic diffraction experiments and using those atomic positions calculated by SIR2011 is shown below (Fig. 8).

The local thickness is responsible for some degree of nonkinematical effects on the experimental pattern such as: first, double diffraction spots and second, the appearance of a few SG-imposed extinctions. These effects have been taken into account in the ED simulation on a second stage. The results are shown in Figure 9.

Purely kinematical effects are always more visible in reflection far from the transmitted beam, whereas the central spots are more sensitive to residual dynamical contributions.

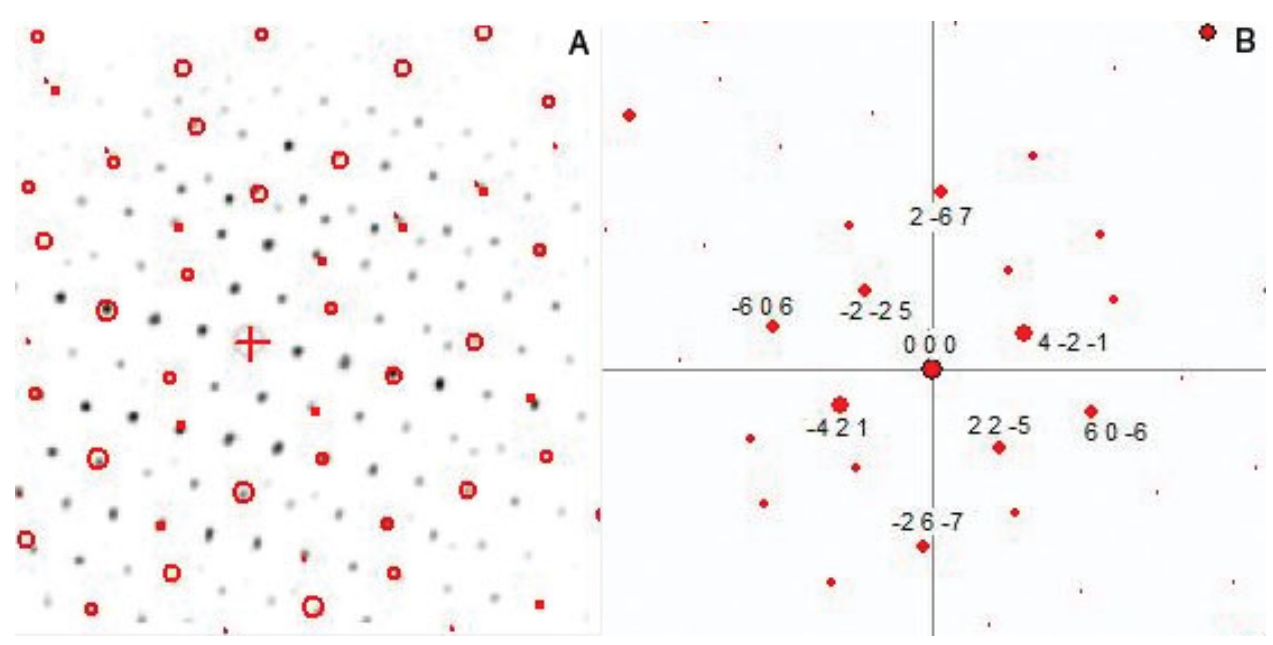

Figure 8. (A) Experimental and simulated patterns overlayed; (B) simulated pattern indexation. 


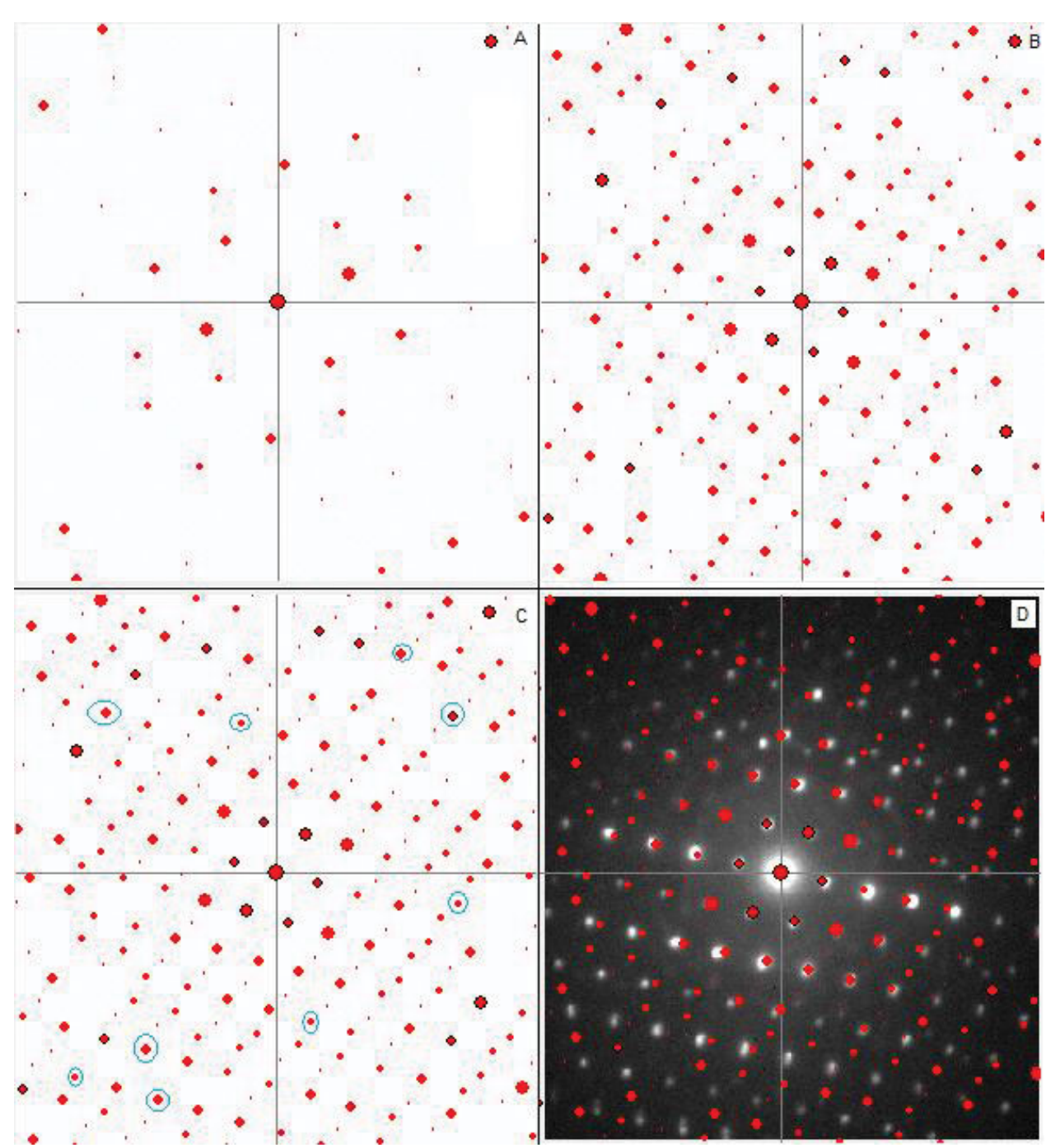

Figure 9. (A) Simulated pattern; (B) central dynamically allowed reflections added to the simulation pattern; (C) double diffraction spots labelled (blue); (D) treated pattern overlayed on the experimental pattern.

\section{CONCLUSION}

It is worth noting that combined results from PED intensities have provided reliable data for the precipitate characterization.

The crystallographic structure of an HfSi precipitate produced from arc-melting iron, $\mathrm{Cr}$, and $\mathrm{Al}$ containing $\mathrm{Hf}$, $\mathrm{Y}$, and $\mathrm{Si}$ (as minor elements) is proposed collecting PED intensities, following the quasi-kinematical approach. The precipitate structure was studied from data with manual 3D diffraction tomography using the direct methods program SIR11. The quality of the available PED data proved sufficient for reliable determination of $\mathrm{Hf}$ and Si positions.

\section{ACKNOWLEDGMENTS}

The authors would like to thank Prof. Edgar Rauch for his valuable comments as well as the CCiT-UB for their microscopy facilities.

\section{REFERENCES}

Burla, M.C., Caliandro, R., Camalli, M., Carrozzini, B., Cascarano, G.L., Giacovazzo, C., Mallamo, M., Mazzone, A.,
Polidori, G. \& Spagna, R. (2012). SIR2011: A new package for crystal structure determination and refinement. J Appl Cryst 45, 357-361.

Gemmi, M., Calestani, G. \& Migliori, A. (2002). Strategies in electron diffraction data collection. Adv Imag Elect Phys 123, 311-325.

Gorelik, T.E., Stewart, A.A. \& Kolb, U. (2011). Structure solution with automated electron diffraction tomography data. Different instrumental approaches. J Microsc 244(3), 325-331.

Kolb, U., Mugnaioli, E. \& Gorelik, T.E. (2011). Automated electron diffraction tomography-a new tool for nano crystal structure analysis. Cryst Res Technol (special issue) 46, 542-554.

Morniroli, J.P. \& Ji, G. (2009). Identification of the kinematical forbidden reflections from precession electron diffraction. $\mathrm{Ma}$ terials Research Symposium Proceedings, p. 1184. Materials Research Society.

Morniroli, J.P., Redjaïmia, A. \& Nicolopoulos, S. (2007). Contribution of electron precession to the identification of the space group from microdiffraction patterns. Ultramicroscopy $107,514-522$.

Otтеn, M.T. (1991). High-angle annular dark-field imaging on a TEM/STEM system. J Elect Microsc Tech 17(2), 221-230.

Own, C.S., Subramanian, A.K. \& Marks, L.D. (2004). Quantitative analyses of precession diffraction data for a large cell oxide. Microsc Microanal 10, 96-104. 
Petricek, V., Dusek, M. \& Palatinus, L. (2006). JanA 2006. The Crystallographic Computing System. Praha, Czech Republic: Institute of Physics.

Rauch, E.F., Véron, M., Portillo, J., Bultreys, D., Maniette, Y. \& Nicolopoulos, S. (2008). Automatic crystal orientation and phase mapping in TEM by precession diffraction. Microsc Anal 22, S5.

Sinkler, W. \& Marks, L.D. (1999). Application of direct methods for crystal structure determination using strongly dynamical bulk electron diffraction. Mater Charact 42(4-5), 283-295.
Vincent, R. \& Midgley, P.A. (1994). Double conical beamrocking system for measurement of integrated electron diffraction intensities. Ultramicroscopy 53, 271-282.

Weirich, T.E., Portillo, J., Cox, G., Hibst, H. \& Nicolopoulos, S. (2006). Ab initio determination of the 393 framework structure of the heavy-metal oxide Csx Nb2.54W2.46O14 from $100 \mathrm{kV}$ precession 394 electron diffraction data. Ultramicroscopy 106, 164-175.

Zou, X., Hovmöller, S. \& Oleynikov, P. (2012). Electron crystallography. Crystallogr Rev 18(4), 253-279. 